\title{
C-06
}

\section{LA PRÁCTICA DE RIEGOS ANCESTRALES ANDINOS: EL RIEGO POR KANIS}

\author{
Moreno-Pérez, M. $F^{(1)}$, Roldán-Cañas, J. ${ }^{(2)}(P)$, Chipana Rivera, $R^{(3)}$ \\ ${ }^{1}$ Profesora Contratada Doctora, Universidad de Córdoba, Dpto. Agronomía (Área Ingeniería \\ Hidráulica), Edif. Leonardo Da Vinci (Campus Rabanales), 14071 Córdoba, mfatima@uco.es \\ ${ }^{2}$ Catedrático, Universidad de Córdoba, Dpto. Agronomía (Área Ingeniería Hidráulica), Edif. \\ Leonardo Da Vinci (Campus Rabanales), 14071 Córdoba, jroldan@uco.es \\ ${ }^{3}$ Profesor, Universidad Mayor de San Andrés, Facultad de Agronomía, Héroes del Acre, \\ 1850, La Paz, Bolivia, renechipana@yahoo.com
}

\section{Resumen}

El riego es una técnica agrícola ancestral desarrollada por las culturas tradicionales andinas, en armonía con las características ambientales y productivas de la zona. La práctica de riego tuvo que ser adaptada a las grandes pendientes dando lugar a técnicas muy particulares que aún hoy día se practican. Los valles interandinos del Departamento de La Paz, Bolivia, es un lugar privilegiado para el estudio de sistemas de riego tradicionales. El trabajo experimental se llevó a cabo en la comunidad de Jatichulaya, localizada en el pueblo de Charazani, Bolivia, a una altitud de $3250 \mathrm{msnm}$ con una precipitación anual media de 450 $\mathrm{mm}$. El sistema de riego tradicional en ella practicado es el llamado riego por kanis. La palabra kanis es un vocablo quechua que hace alusión a la apertura de surcos para la conducción del agua. El sistema de riego no causa erosión del suelo ya que el manejo del agua se hace siguiendo las curvas de nivel. Sin embargo, las necesidades de mano de obra son muy elevadas. La persona a cargo de la distribución del agua y de su entrega a los regantes es el llamado Agente de Aguas.

\begin{abstract}
Irrigation is an ancient agricultural technique developed by the Andean traditional cultures in harmony with environmental and productive characteristics of the area. Irrigation practice had to be adapted to the steep slopes leading to very specific techniques that are practiced even today In Bolivia, the interandean valleys of La Paz's Department are privileged places for the study of traditional irrigation systems. The experimental work was carried out in the peasant community of Jatichulaya, located in Charazani, at an altitude of 3,250 meters above sea level, with an average annual rainfall of about $450 \mathrm{~mm}$. The traditional irrigation system practiced there is called the kanis's method. The word kanis is a quechua word that refers to furrow irrigation opening for water conveyance. This irrigation system avoids soil erosion because it follows contour lines. Thereby, soil is protected but manpower required in the irrigation practice was excessive. The person in charge of water distribution and delivery to irrigators is called Agente de Aguas (Water's officer).
\end{abstract}

\section{1- Introducción y Objetivos}

En Bolivia la superficie total bajo riego es de unas 303000 ha, el $65 \%$ de las cuales se localiza en el Altiplano y en los valles interandinos, entre los 1500 y los 4200 msnm (Ministerio de Medio Ambiente y Agua, 2013). En estas zonas la pluviosidad anual se sitúa entre los 350 y los $600 \mathrm{~mm}$ que cae, principalmente, entre los meses de diciembre y marzo. 
A pesar de las grandes pendientes y del accidentado terreno, el riego mayoritario es por superficie, y el $70 \%$ de los regantes poseen menos de 1 ha.

En los valles mesotermales existentes en las cordilleras andinas se desarrollaron culturas prehispánicas muy florecientes, como la cultura Mollo (Ponce Sanginés, 1957, Arellano López, 1978) que transformaron la abrupta geografía para poder cultivarla. Los mollos desarrollaron técnicas para construir terrazas, tanques de almacenamiento de agua en rocas, canales para conducir agua de formas trapezoidales y triangulares y estructuras de captación de agua (Chipana et al., 2011). El riego es una técnica agrícola ancestral desarrollada por las culturas tradicionales andinas, en armonía con las características ambientales y productivas de la zona. La práctica de riego tuvo que ser adaptada a las grandes pendientes dando lugar a técnicas muy particulares, como el riego por kanis o los surcos corrugados en zigzag (Roldán-Cañas et al., 2014), que aún hoy día se efectúan en zonas próximas a la ciudad de La Paz, Bolivia. Los campesinos lograron, durante siglos, aprovechar las aguas de los ríos, lagunas y manantiales para complementar o suplir el régimen de precipitaciones pluviales, caracterizado por su irregular distribución en una corta estación de lluvias.

La palabra kanis es un vocablo quechua que hace alusión a la apertura de surcos para la conducción del agua.El riego por kanisconsta de unos canales principales de distribución llamados mama kanis, cuya longitud y número dependen del tamaño de la parcela a regar. A continuación, se encuentran los canales secundarios o juchuy kanis que van en dirección opuesta a los mama kanis. De los juchuy kanis parten los surcos de riego de corta longitud. El riego se empieza en el extremo más alejado y se va desplazando hacia atrás. La particularidad de este riego radica en la forma en que se practica pues los canales y surcos se abren conforme el agua va entrando en la parcela. Ello supone, por un lado, un tamaño de parcelas relativamente pequeño y, por otro lado, un alto requerimiento de mano de obra. Por el contrario, cabe esperar una uniformidad muy alta, dada la pequeña longitud de los surcos de riego, y un mayor control en la distribución del agua evitando pérdidas (Chipana Rivera et al., 2015). Es curioso comprobar que este sistema presenta grandes similitudes con los sistemas de riego árabes en forma de peine tal y como son descritos por del Pino et al. (2011).

El objetivo principal de este trabajo es analizar el funcionamiento, eficiencia y sostenibilidad del riego por kanis.

\section{2- El valle de Charazani y la comunidad de Jatichulaya}

Charazani -o Villa Juan José Pérez-, capital del valle al que da nombre, se encuentra situado a una altitud de $3250 \mathrm{msnm}$, en la Provincia Bautista de Saavedra, Departamento de La Paz, Bolivia (ver figura 1). Abarca una superficie de $2524 \mathrm{~km}^{2}$ y está constituida por dos secciones municipales, Charazani y Curva, y 14 cantones. Conforma el espacio sociogeográfico más significativo para el estudio del riego tradicional ya que el agua en la zona es un recurso escaso del que dependen las actividades agrícolas que realiza el campesinado. En efecto, la precipitación media anual oscila alrededor de los $450 \mathrm{~mm}$ distribuidos principalmente entre los meses de diciembre a marzo.

Aquí vive, entre otras, la comunidad campesina de Jatichulaya, situada entre los paralelos de $15^{\circ} 8^{\prime}$ y $15^{\circ} 12^{\prime}$ de Latitud Sur, y los meridianos de $68^{\circ} 57^{\prime}$ y $68^{\circ} 59^{\prime}$ de Longitud Oeste, y una altitud entre los 2900 a 3250 m.s.n.m (ver figura 2). En la actualidad, posee una superficie aproximada de 202 ha, de las que 45.5, 22.5\%, cuentan con riego siendo el resto tierras de secano. La zona presenta una topografía accidentada, con laderas de pendientes empinadas y terrazas angostas. Los suelos son de tipo coluvial, variando la textura entre franco arcilloso y franco arenoso. 


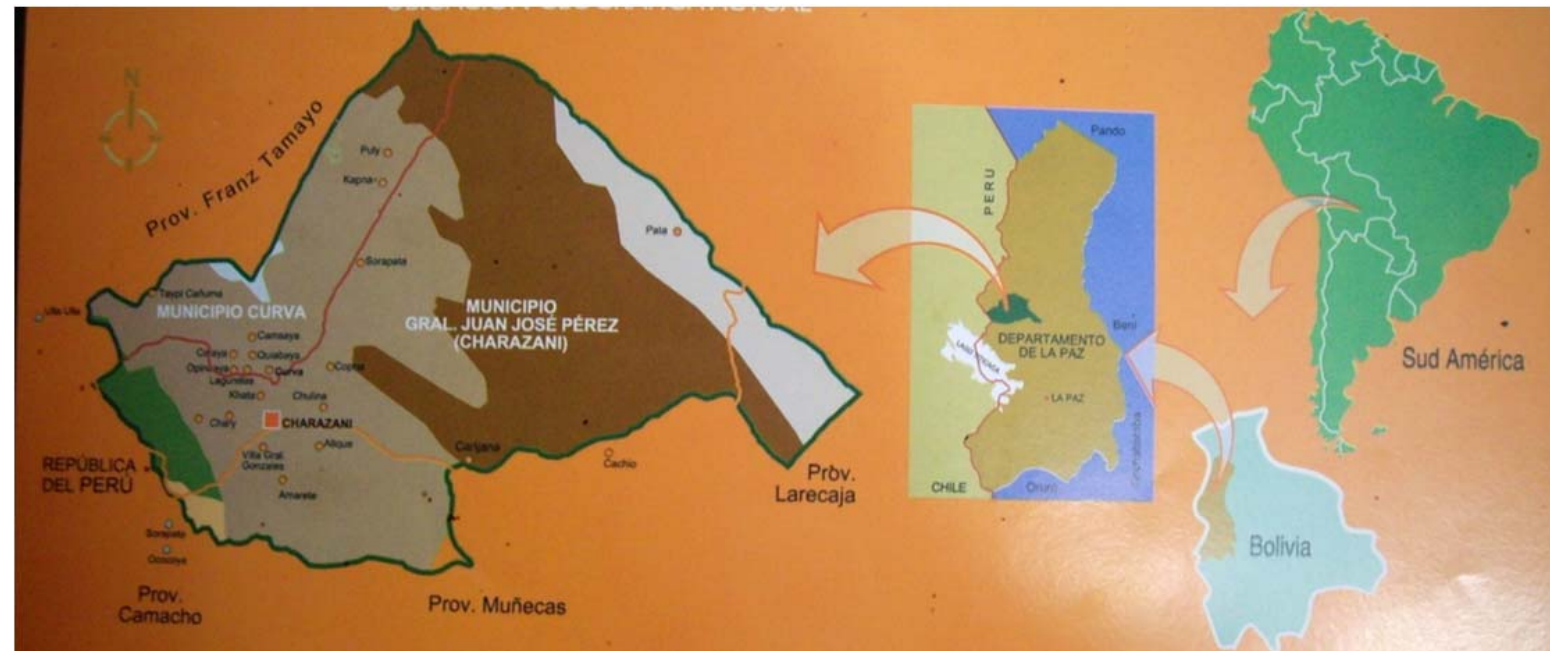

Figura 1. Ubicación del municipio de Charazani dentro del Departamento de La Paz.

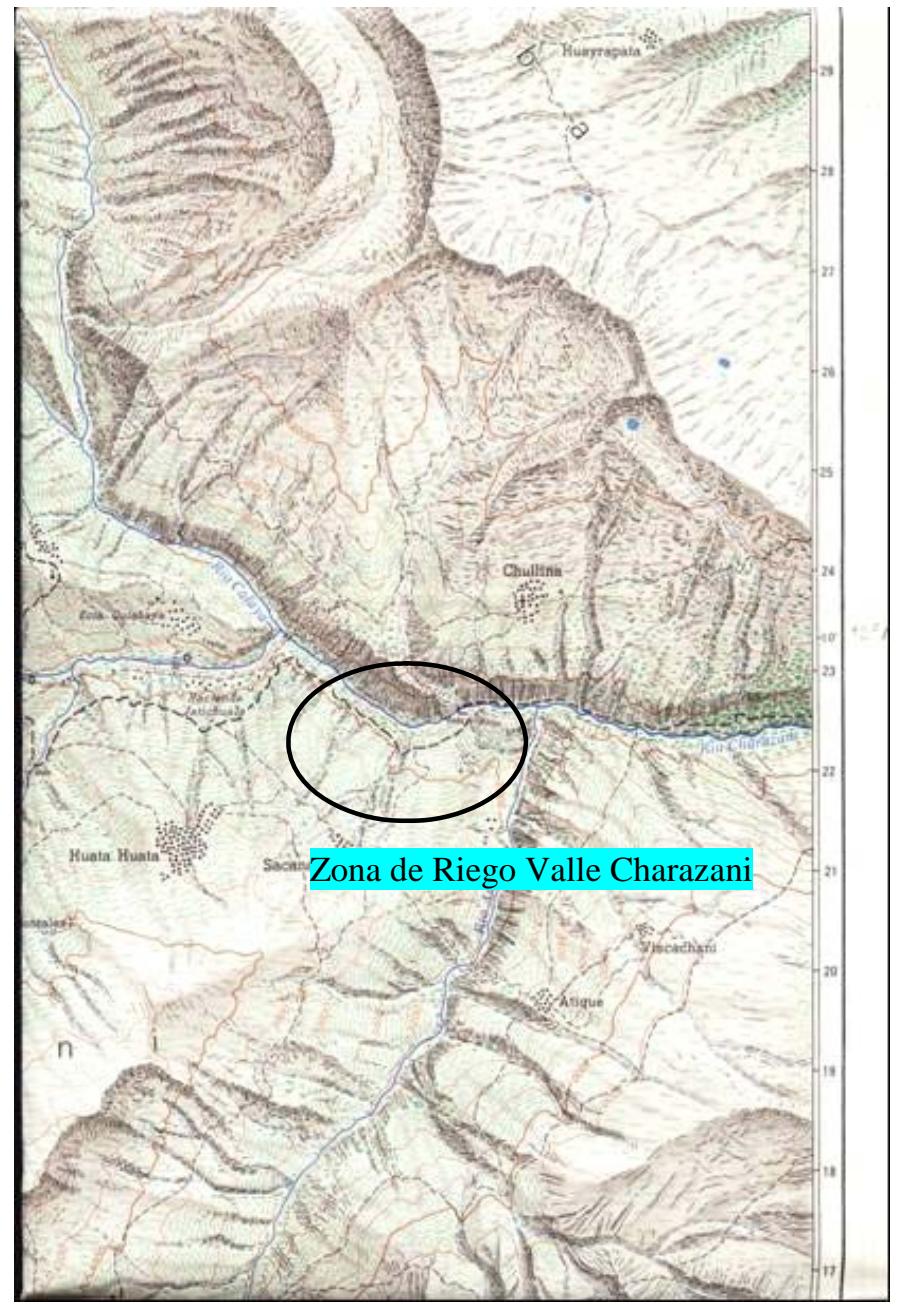

Figura 2. Ubicación de la comunidad de Jatichulaya.

Las fuentes de agua son las precipitaciones pluviales y las aguas superficiales, producto del deshielo de la cordillera de Apolobamba, que dan origen al Río Calaya y a parte del Río Charazani. El Río Charazani es una de las fuentes de agua de importancia para la zona de estudio y tiene su origen en las pequeñas vertientes y en los llamados ojos 
de agua (aguas subterráneas). También existen pequeñas vertientes situadas al sureste de la comunidad de Charazani, que forman el Río Wasawayqho, con caudales reducidos en épocas de estiaje, de mayo a julio, que aumentan en la época de lluvias. Existe otro ojo de agua en la parte baja de la comunidad de Inca Roca, situado al Sur de la comunidad de Jatichulaya (Quebrada del Inca), originando de esta manera la tercera fuente de agua para la comunidad.

\section{3- Organización del riego}

El manejo del agua está de acuerdo a los ciclos agrícolas que se practican en el valle de Charazani. La distribución del agua de riego varía a lo largo del año. Desde agosto hasta enero o febrero, se hace bajo un control estricto del llamado Agente de Aguas y el establecimiento de un turno específico cuya trasgresión es sancionada por el mismo. El Agente de Aguas es elegido anualmente y dentro de sus funciones, que hace mediante visitas periódicas, están, además, el mantenimiento y cuidado de todas las obras hidráulicas que posee el sistema, es decir, las obras de toma, canales de conducción y de distribución (Céspedes, 1995). El sistema de distribución consiste en programar el uso del agua de riego de acuerdo a los pedidos que hagan los usuarios en un período dado. El agua se entrega de acuerdo al turno programado por el Agente de Aguas y el usuario tiene que ajustarse a dicho turno. La adopción de la distribución por turnos comienza en el mes de agosto con la siembra de papa-milli (papa temprana), cultivo que requiere de riego. El Agente de Aguas lo hace en forma equitativa sin dar preferencia a nadie.

Por el contrario, en la época donde no existe mucha demanda de agua de riego ya que los cultivos satisfacen, en gran manera, su requerimiento hídrico con las precipitaciones pluviales, esto es, desde febrero hasta julio, el uso del agua no es por turnos sino a la demanda y las personas que deseen regar piden permiso, o bien dan aviso al Agente de Aguas, y van ellos mismos hasta la toma conveniente a soltar el agua.

Los campesinos organizan las asambleas, presididas por el Agente de Aguas, donde planifican y se establecen las fechas fijas para los trabajos comunales de reparación y limpieza de canales. Los trabajos de limpieza incluyen también la reparación de los caminos de herradura usados para el manejo del agua. Estos caminos van paralelos al recorrido de los canales. Las fechas de los trabajos de limpieza generalmente son antes del inicio de la siembra grande, normalmente en el mes de agosto.

\section{4- La práctica del riego}

El riego comienza con la construcción de un canal de reparto de agua dentro de la parcela. Los principales indicadores para la construcción de dicho canal son, por un lado, la entrada del agua desde el canal de distribución a la parcela y, por otro lado, la pendiente de la misma. Una vez que se tienen los dos parámetros, se traza una línea imaginaria hasta el otro extremo superior de la parcela de modo que tenga una pendiente descendente del 2 al $3 \%$. Una vez trazada dicha línea imaginaria se procede a la apertura del canal con la ayuda de una Laukana (ver figura 3), de abajo hacia arriba hasta llegar al extremo inicial o parte superior del canal. El canal tiene la forma de un surco amplio y es de tierra.

La longitud y el número de canales principales o mama kanis es variado y está en función del tamaño de la parcela a regar lo que constituye el tercer parámetro para la construcción de los canales. Generalmente, como mínimo, se construyen 2 o 3 canales. La separación entre los canales construidos es de 8 a $10 \mathrm{~m}$. Cuando el terreno es accidentado, o con una topografía irregular, el campesino consigue trazar los canales principales sin dificultad empleando todo el conocimiento ancestral heredado de sus padres y abuelos. La construcción de los mama kanis dentro de la parcela es similar sea cual fuere el cultivo. 


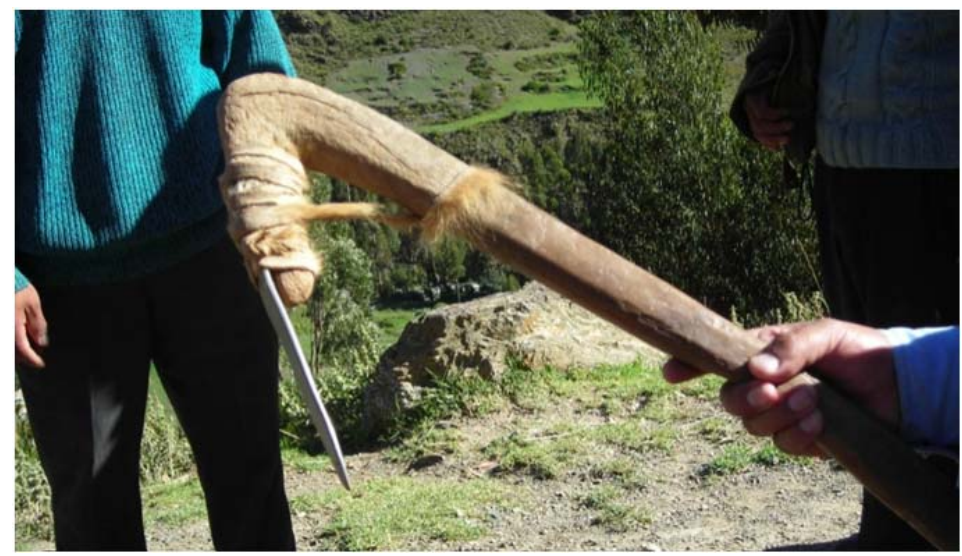

Figura 3. Laukana o instrumento utilizado para abrir o cerrar los surcos de riego.

La orientación de las parcelas es importante, ya que las que se encuentran orientadas al sur no tienen muro de contención, y además se riegan, mientras que las orientadas al norte tienen muro de contención y no hay que regarlas. Se observa como las parcelas orientadas al norte son más verdes que las orientadas al sur que son más secas.

Para evaluar la práctica actual de riego, se realizó un ensayo en una parcela previamente preparada y desbrozada, situada a $3065 \mathrm{msnm}$, en la que la comunidad de Jatichulaya hizo una demostración de las prácticas de riego habituales en la zona. Esta parcela tiene una extensión aproximada de $150 \mathrm{~m}^{2}$ y una pendiente que oscila entre $15 \mathrm{y}$ el $45 \%$. Según estimaciones de la propia Comunidad, el canal de abastecimiento de agua o kani suele llevar entre 4 y $5 \mathrm{l} / \mathrm{s}$, y el tiempo que se invierte en regarla es de unos $15 \mathrm{~min}$. En la parcela trabajada por los campesinos, el mama kani divide el terreno en dos. En este caso, se procede al riego de la mitad inferior.

El campesino inicia la apertura de un mama kanis para que el agua procedente de un kanis entre a la parcela y la inunde (ver figuras 4a y b). Para la construcción de los canales secundarios o juchuy kanis dentro de la parcela se toma al canal principal, como referencia. Los juchuy kanis generalmente van en dirección opuesta a los mama kanis, son paralelos entre si y tienen también inclinaciones que están entre el 2 y el $3 \%$. La longitud de los juchuy kanis es menor con relación a la de los mama kanis y tienen, como promedio, de 6 a $8 \mathrm{~m}$. En cambio, el número de los juchuy kanis es mayor, superior a 6 canales habitualmente. La separación entre juchuy kanis es de 4 a $4.5 \mathrm{~m}$. como promedio y son también surcos aunque más angostos que los canales principales. Una de las funciones de los juchuy kanis es la de unir los mama kanis, cuando el usuario está conduciendo el agua hacia la parte baja de la parcela.
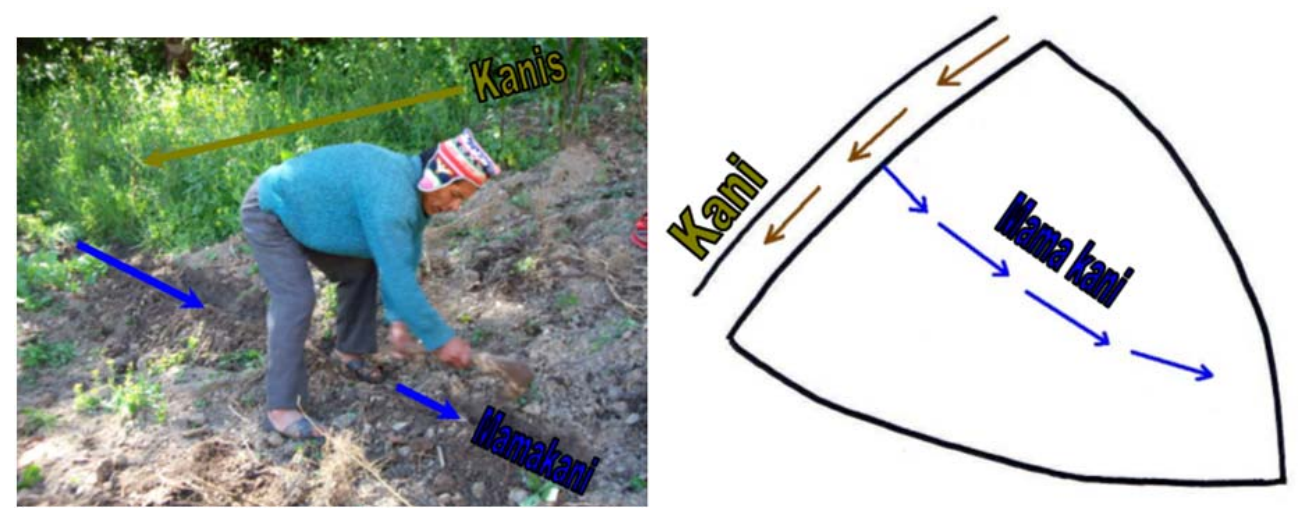

Figura 4. a) Campesino iniciando la apertura de un mama kanis. b) Esquematización de 4a. 
Cuando el agua llega al final del mama kani, se van abriendo juchuy kanis desde el final del mama kani hacia atrás, para que el agua humedezca la margen derecha del mama kani (ver figura 5). Mientras el agua va empapando el suelo de la parte final de la parcela, con cuidado de que no rebose y caiga a la parcela que hay por debajo, se abre un nuevo surco que parte desde el mama kani por su parte central, y que será otro mama kani, para que cuando los juchuy kanis lleguen a éste, se derive todo el agua hacia el nuevo mama kani. El sistema funciona apropiadamente, acomodándose a las condiciones topográficas de la zona y eliminando la erosión hídrica debido a que el manejo del agua, dentro y fuera de la parcela, está dado por un conocimiento ancestral de los campesinos de acuerdo a las curvas a nivel, lo que permite la protección del suelo sin provocar erosión.

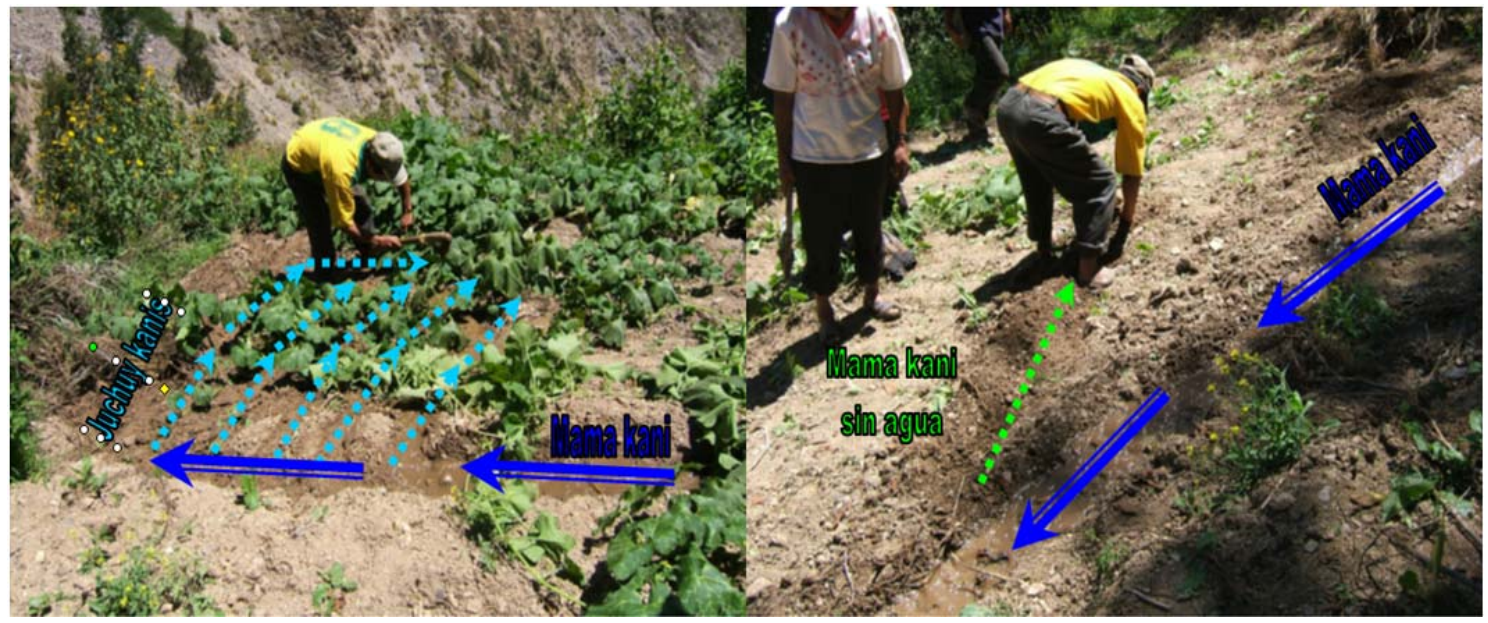

Figura 5. Apertura de juchuy kanis desde el final del mama kani.

Cuando los juchuy kanis han llegado hasta el segundo mama kani $(\longrightarrow$ ), que parte desde el primer mama kani ( $($ ), se abre el paso de agua hacia este quedándose sin agua el trozo del primer mama kani situado aguas abajo ( $\Longrightarrow$ ) (ver figuras $6 a$ y b).
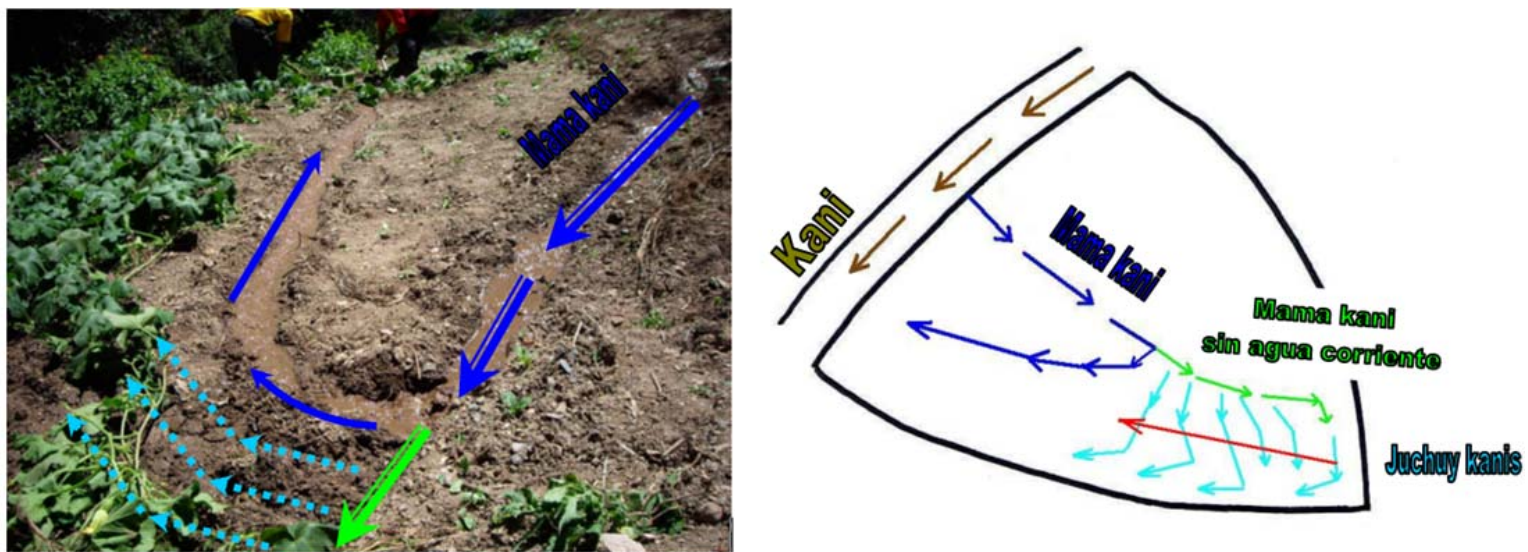

Figura 6. a) Al abrir un nuevo mama kani, los juchuy kanis aguas arriba se quedan sin agua.

b) Esquematización de la figura $6 a$.

Cuando el agua ha llegado al final del segundo mama kani se empiezan a abrir juchuy kanis desde el final y con pendiente descendente (ver figuras7a y $7 \mathrm{~b}$ ). 

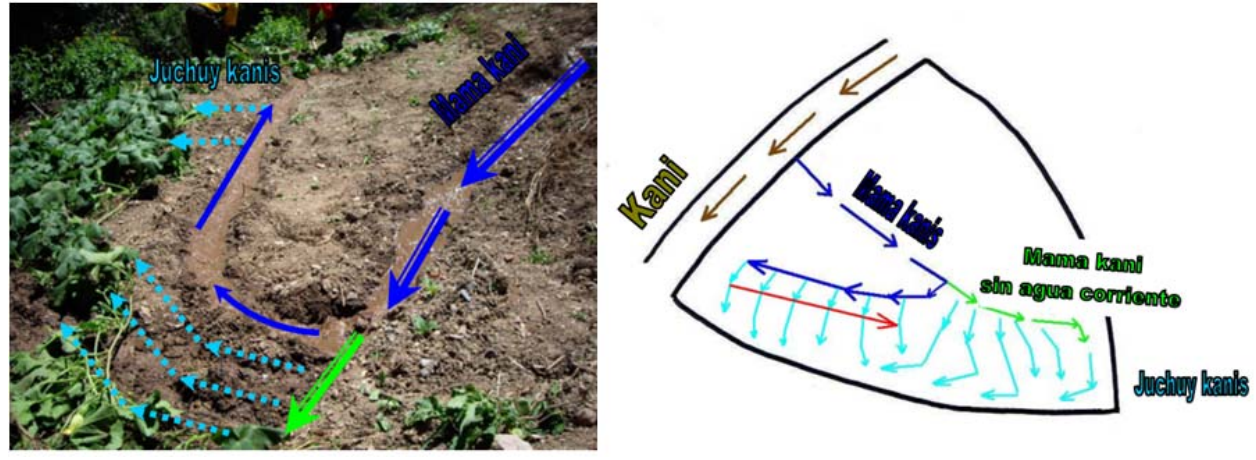

Figura 7. a) Apertura de los juchuy kanisdesde el final del segundo mama kani y con pendiente descendente. b) Esquematización de la figura $7 a$.

Finalmente, la mitad de la parcela quedó regada (ver figuras $8 a$ y b). El tiempo invertido fue de unos 40 minutos, muy superior al manifestado previamente por la Comunidad.
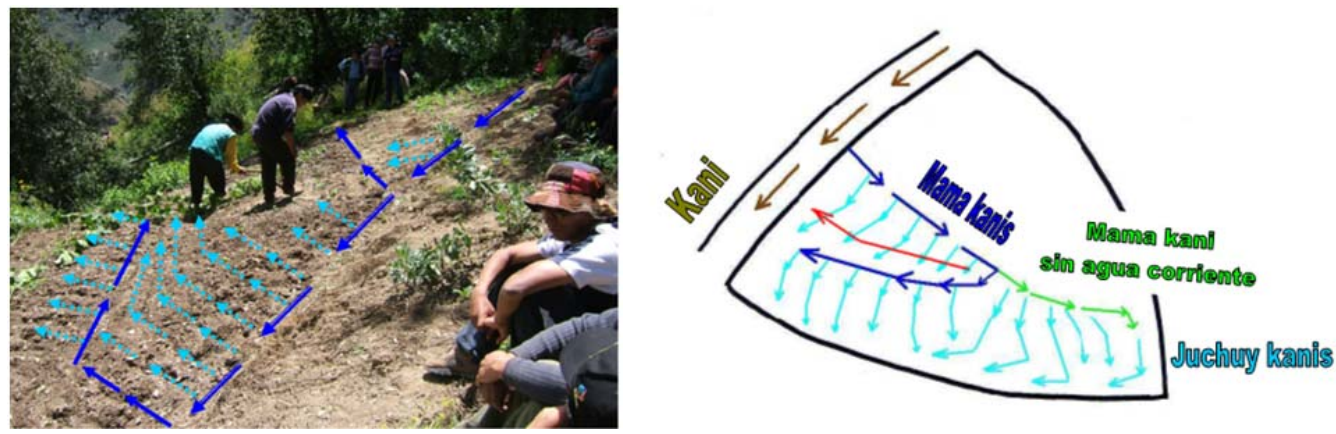

Figura 8. a) Fase final del riego. b) Esquematización de la figura 8a.

Una vez que se terminó el riego de la parcela, se midió el caudal que lleva el canal de riego, utilizando el método del flotador con una hoja seca. La medida se realizó repetidas veces tomándose la media de los valores obtenidos: $L=2.5 \mathrm{~m} ; \mathrm{t}=7 \mathrm{~s}$.

Teniendo en cuenta la geometría de la sección transversal del canal rectangular( $\mathrm{B}=$ $0.25 \mathrm{~m} ; \mathrm{y}=0.036 \mathrm{~m})$ ) el caudal circulante resulta ser de:

$$
Q=v \cdot \omega=\frac{2.5 \mathrm{~m}}{7 \mathrm{seg}} \cdot 0.25 \mathrm{~m} \cdot 0.036 \mathrm{~m}=3.2 \mathrm{l} / \mathrm{seg}
$$

valor parecido al que los campesinos comentaron previamente. No se ha aplicado un coeficiente reductor en el cálculo del caudal dado el escaso calado de la corriente.

Conocido el tiempo invertido (40 minutos) y la superficie regada $\left(75 \mathrm{~m}^{2}\right)$, se pueden calcular las características agronómicas del riego:

Volumen aplicado: $3.2 \mathrm{l} / \mathrm{s} \times 40 \times 60 \mathrm{~s}=7680$ litros

Dosis por riego $=7680$ litros $/ 75 \mathrm{~m}^{2}=102.4 \mathrm{l} / \mathrm{m}^{2} \approx 1000 \mathrm{~m}^{3} / \mathrm{ha}$

Dado que en una campaña normal de riegos se aplican 4 riegos, el volumen total aplicado es de $4000 \mathrm{~m}^{3} / \mathrm{ha}$, bien ajustado a las características de la zona. No obstante, la mano de obra requerida se aleja del óptimo, ya que se necesitó del concurso de 3 personas. Posiblemente, nuestra presencia y la necesidad de explicar a la misma vez el procedimiento de riego aumentaron tanto el tiempo de riego como la mano de obra utilizada. 


\section{5- Conclusiones}

En Bolivia, los valles interandinos de La Paz constituyen lugares privilegiados para el estudio de sistemas de riego tradicionales, por el importante legado de los campesinos andinos, que durante siglos, lograron aprovechar las aguas de los ríos, lagunas y manantiales para satisfacer sus necesidades, dentro de la cosmovisión holística del mundo que construyeron, en la que el agua tenía un papel fundamental.

En este estudio se ha tratado de describir y comprender los sistemas tradicionales de riego, mediante la descripción de infraestructuras y la caracterización de la organización en la distribución de las aguas. El trabajo de campo se desarrolló en la Provincia Bautista Saavedra del Departamento de La Paz donde se analizó el sistema de riego tradicional practicado por comunidades campesinas pertenecientes a la municipalidad de Charazani en áreas de dominio de las comunidades de Jatichulaya, donde el riego está condicionado al método de kanis.

Este método consiste en un riego superficial, ampliamente desarrollado por las culturas tradicionales andinas del país, completamente adecuado y en equilibrio con las características ecológicas y productivas de la zona. No obstante, se pudo evidenciar un elevado uso de mano de obra en la práctica del riego quizás derivado de nuestra presencia y de la necesidad de explicarnos a la vez el procedimiento de trabajo.

\section{6- Agradecimientos}

Este trabajo se desarrolló en el marco de proyectos desarrollados entre 2008 y 2009 y financiados por la Agencia Española de Cooperación Internacional para el Desarrollo (AECID), a quién se agradece su colaboración, y en el que participaron profesores de las Universidades de Córdoba, Córdoba, España, y Mayor de San Andrés, La Paz, Bolivia.

\section{7- Bibliografía}

Arellano López, J. (1978). La cultura Mollo: ensayo de síntesis arqueológico. INAR, La Paz, $81 \mathrm{p}$.

Céspedes, R., (1995). Descripción y Análisis del Sistema de riego Tradicional en la Comunidad de Jatichulaya de la provincia Bautista Saavedra.Tesis de Grado Universidad Mayor de San Andrés - La Paz, 140 p.

Chipana, R., Bosque, H., Del Pino, J.L., Roldán-Cañas, J. \& Moreno-Pérez, M.F.(2011).Sistemas de riego prehispánicos en Bolivia. En: Roldán-Cañas, J. \& Chipana, R. (coords.), 2011. Sistemas ancestrales de riego a ambos lados del Atlántico. Universidad de Córdoba, España, pp. 1-61.

Chipana Rivera, R., Moreno-Pérez, M.F., Choque Velasco, R.\& Roldán-Cañas, J. (2015). Performance of pre-hispanic irrigation systems in the Andean región of Bolivia. Agricultural Water Management,Published online 27/11/2015,DOI: http://dx.doi.org/10.1016/j.agwat.2015.11.010.

Del Pino, J.L., Roldán-Cañas, J. \& Moreno-Pérez, M.F.(2011). El agua y el riego en laPenínsula Ibérica. En: Roldán-Cañas, J. \& Chipana, R. (coords.), 2011. Sistemasancestrales de riego a ambos lados del Atlántico. Universidad de Córdoba, España, pp. 63-232. 
Ministerio de Medio Ambiente y Agua. (2013). Inventario Nacional de Sistemas de Riego. Bolivia, $29 \mathrm{p}$.

Ponce Sanginés, C. (1957). La cerámica Mollo. Biblioteca Paceña, La Paz.

Roldán-Cañas, J., Chipana, R., Moreno-Pérez, M. F. \& Chipana, G. (2015). Description and Evaluation of Zigzag Furrow Irrigation in the Inter-Andean Valleys of Bolivia. Journal of Irrigation and Drainage Engineering, 141(11): 04015019, DOI: 10.1061/(ASCE)IR.1943-4774.0000902. 